\title{
Os benefícios do Mindfulness para um envelhecimento saudável e sustentável
}

Mindfulness benefits for a healthy and sustainable aging

\section{Diana Pinheiro}

WFCMS - World Federation of Chinese Medicine Societies

dianapinheiro.saude@gmail.com

\section{Manuela Rodrigues}

Egas Moniz Cooperativa de Ensino Superior, Portugal

mindfuldentistry@gmail.com

Conflito de interesses: nada a declarar. Financiamento: nada a declarar.

Data de Submissão: 12/06/2021

Data de Aprovação: 29/07/2021

Todo o conteúdo do JIM é licenciado sob Creative Commons, a menos que especificado de outra forma e em conteúdo 
https://doi.org/10.29073/jim.v2i2.426

\section{RESUM0:}

Introdução: 0 envelhecimento é inevitável, contudo envelhecer não significa necessariamente ausência de saúde. Normalmente, as medidas que promovem um envelhecimento saudável focam nos comportamentos físicos, alimentares e de estilo de vida, que podem permitir envelhecer com saúde. 0 mindfulness, tem benefícios biológicos, psicológicos e sociais comprovados, sendo por isso também de considerar, para melhor compreender e gerir algumas das frequentes condições na saúde associadas ao envelhecimento.

Objetivos: 0 objetivo desta revisão é fornecer uma visão panorâmica sobre o que é o mindfulness, quais os benefícios estudados e como pode contribuir com estratégias práticas e eficazes para um envelhecimento saudável e sustentável.

Metodologia: Fez-se revisão de 33 artigos publicados entre 2001 e 2021, com envolvimento total de 129 autores nos respetivos artigos, com acesso a 130 estudos. A pesquisa relativamente aos 33 artigos foi realizada nos bancos de dados Researchgate e PubMed e permitiu identificar estudos que investigam os efeitos e benefícios do Mindfulness, técnicas e sistemas e programas associados, em adultos com idade superior a 55 anos. As bases de dados consultadas pelos respectivos autores dos artigos de revisões simples e sistemáticas com meta-análise foram a CABI, Cinahl, Cochrane, Embase, Google Scholar, Medline, Pschyline, Psychlnfo, PubMED e Web of Science.

Conclusão: 0 Mindfulness, as técnicas que o cultivam e suportam, como a meditação, a respiração e 0 movimento mindful, contribuem significativamente para um envelhecimento mais saudável e sustentável.

Área de investigação: Gerontologia, Mindfulness, Bem-Estar, Técnicas e estratégias para idosos

Palavras-chave: Envelhecimento Saudável, Mindfulness, Meditação, Idosos 
https://doi.org/10.29073/jim.v2i2.426

\section{ABSTRACT:}

Introduction: Aging is inevitable; however, it does not necessarily have to imply lack of health. Usually, the measures that promote healthy aging focus on physical, dietary and lifestyle behaviors that can allow a person to age in a healthier way. Mindfulness has proven biological, psychological, and social benefits, which is why it is also important to consider it to better understand and manage some of the frequent health conditions associated with aging.

Objectives: The aim of this review is to provide an overview of what mindfulness is, what benefits it has studied and how it can practically and effectively contribute to healthy and sustainable aging.

Methods: 33 references published between 2001 and 20201 were reviewed, 129 authors with access to 130 studies. We searched the Researchgate and PubMed databases to identify studies investigating the effects and benefits of Mindfulness in adults over 55 years of age.The databases consulted by the respective authors of simple and systematic review articles with meta-analysis were CABI, Cinahl, Cochrane, Embase, Google Scholar, Medline, Pschyline, Psychlnfo, PubMED and Web of Science.

Conclusion: This review highlights Mindfulness, and the techniques that cultivate and support it, such as meditation and mindful movement, to significantly contribute to a healthier and more sustainable aging.

Subject Areas: Gerontology, Mindfulness, Well-Being, Techniques and Strategies for the Elderly

Keywords: Mindfulness, Meditation, Healthy Aging, Elder People 


\section{REVISÃO DE LITERATURA}

\subsection{Introdução}

0 consenso geral de que as habilidades cognitivas tendem a diminuir com o avanço da idade é apoiado por vários estudos que relataram que os adultos mais velhos têm um desempenho mais baixo em vários testes de desempenho cognitivo em comparação com os adultos mais jovens (Prakash, Rastogi, Dubey, Abhishek, Chaudhury, Small, 2012). Com uma sociedade que envelhece rapidamente, torna-se cada vez mais importante compreender e gerir o declínio normal do funcionamento cognitivo relacionado com a idade (Gard, Hölzel, Lazar, 2014). As intervenções baseadas em mindfulness oferecem uma abordagem de tratamento articulado, holístico mente - corpo e complementar baseada em evidências (HazlettStevens, Singer, Chong, 2019), podendo atuar nas três dimensões do ser humano, Bio-Psico-Social (Nilsson, Bülow \& Kazemi, 2015).

\subsection{0 que é 0 Mindfulness}

Germer, C. (2004) afirma que "Mindfulness," é uma tradução para o inglês da palavra "Sati", que significa "Atenção Plena" em Pali, a língua em que os sábios e ancestrais ensinamentos de Buda foram originalmente registados.

A primeira tradução do dicionário de "Sati" para "Atenção Plena" data de 1921 (Davids \& Stede, 1921/2001).

Figura 1. Definições de "Mindfulness"

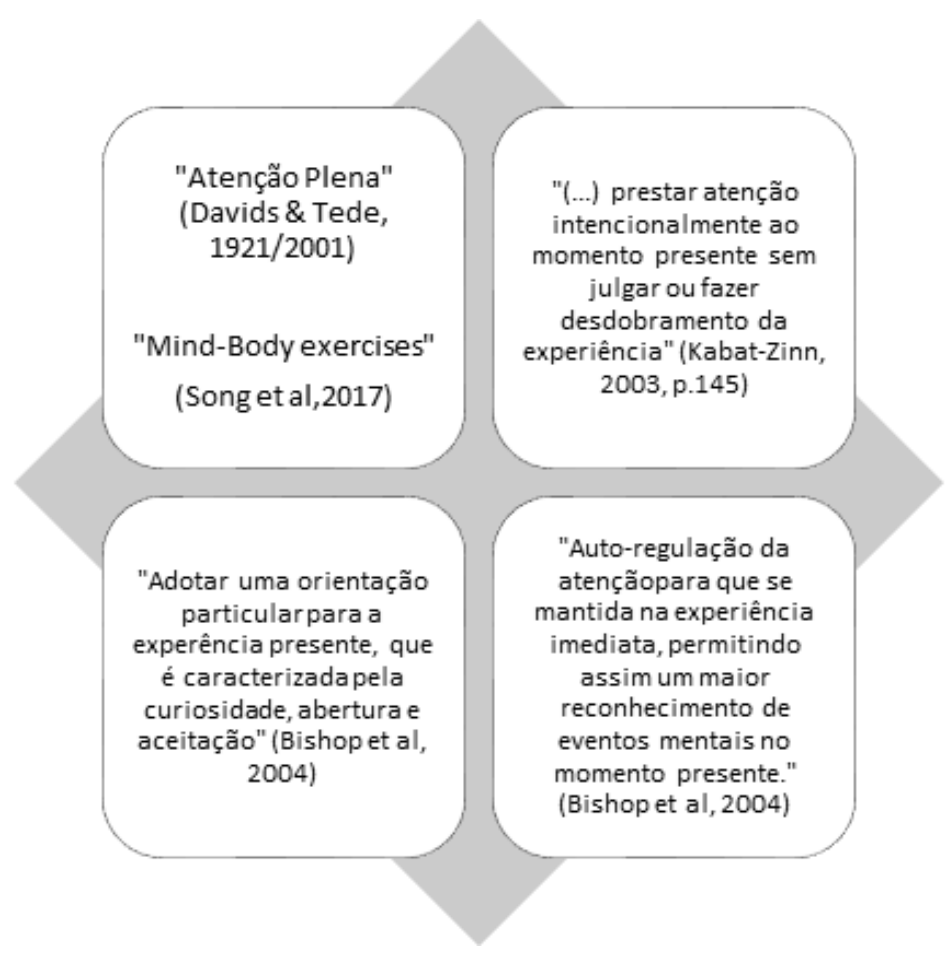


https://doi.org/10.29073/jim.v2i2.426

Fonte: Elaboração própria com base nos autores citados

A definição de Davids \& Stede, tem vindo a ser ampliada de forma a permitir uma maior compreensão e conferir uma melhor acessibilidade, compreensão e aplicabilidade deste treino, que alia foco mental a movimento respiratório e corporal, dirigido e adaptado a diferentes contextos: psicoterapêuticos, bemestar pessoal, empresarial, escolar e académico, bem-estar e saúde gerais. Tem vindo também a ser implementado amplamente, nas últimas décadas, em instituições públicas e privadas dedicadas à população sénior, nesta com o forte e focado objectivo não só ao nível do seu bem-estar geral, mas também no que diz respeito à redução do número de quedas sendo que por exemplo prática mindful do Tai Ji tem provado ser eficaz (Harmer, Eckstrom, Fitzgerald, Chou, \& Liu, 2019) os autores monitorizam 1147 pessoas com idade média de 77.7. Os resultados primários foram a incidência de quedas prejudiciais moderadas e graves em 12 meses, medidas como taxas de taxa de incidência.

\subsection{Práticas mindful - de atenção plena - dentro do Mindfulness}

\begin{tabular}{|l|l|}
\hline \multicolumn{1}{|c|}{ Práticas Mindful } & \multicolumn{1}{|c|}{ Descrição } \\
\hline Meditação sentada & $\begin{array}{l}\text { Sentado, relaxado e alerta, por vários minutos, apenas "sendo" e } \\
\text { experimentando a quietude. Praticar ter consciência de } \\
\text { pensamentos, emoções e sensações com uma mente curiosa, } \\
\text { aberta, compassiva e sem julgamentos }\end{array}$ \\
\hline Consciência da respiração & $\begin{array}{l}\text { Direcionar suavemente o foco de atenção para a respiração } \\
\text { enquanto inspira e expira naturalmente }\end{array}$ \\
\hline Meditação a andar & $\begin{array}{l}\text { Notar a atenção dos pés ao contactar com a superfície a cada passo } \\
\text { e percebendo as sensações no corpo. }\end{array}$ \\
\hline Agradável / Desagradável & $\begin{array}{l}\text { Perceber com interesse, abertura e curiosidade as sensações físicas } \\
\text { e emocionais ao nosso redor. }\end{array}$ \\
\hline $\begin{array}{l}\text { Meditações guiadas pelos } \\
\text { sentidos }\end{array}$ & $\begin{array}{l}\text { Ter atenção de maneira intencional ao que ouvimos, tocamos, } \\
\text { cheiramos, saboreamos e / ou observando de uma forma aberta, } \\
\text { sem julgamentos e expansiva. }\end{array}$ \\
\hline Body Scan & $\begin{array}{l}\text { Notar lenta e intencionalmente o corpo. 0 foco está em estar } \\
\text { relaxado e consciente enquanto criamos uma conexão (ao invés de } \\
\text { resistência) com o corpo e as diversas sensações nele presente. }\end{array}$ \\
\hline
\end{tabular}


https://doi.org/10.29073/jim.v2i2.426

\begin{tabular}{|c|c|}
\hline $\begin{array}{lr}\text { MindBody } & \text { exercises } \\
\text { Movimento } & \text { consciente: } \\
\text { Yoga (origem indiana); Qi } \\
\text { Gong e Tai Ji (Origem } \\
\text { Chinesa) }\end{array}$ & $\begin{array}{l}\text { A integração da mente e corpo por meio da respiração, posturas, } \\
\text { fluxo, movimento e atenção plena. }\end{array}$ \\
\hline
\end{tabular}

A prática de mindfulness consiste assim na prática de técnicas ou movimento "mindful" também designados de "Mind-body exercises" (Song, Grabowska, Park, Osypiuk, et al, 2017) e podem incluir: desde práticas de exercícios isolados tais como a meditação, atenção consciente e plena à respiração, às sensações corporais, emoções ou exercícios que são sugeridos de forma guiada e integrada dentro de programas tais como o Mindfulness Based Stress Reduction (MBSR), ou dentro de sequências de exercícios tais como a ginástica terapêutica ou meditação em movimento "Yoga", "Qi Gong" e "Tai Ji".

Tabela 1. As diferentes técnicas, práticas e sistemas Mindful

Fonte: Elaboração própria adaptado de Kebat-Zinn (2013) e Song, et al (2017)

A Respiração consciente, ou a atenção plena à respiração é uma das técnicas utilizadas no Mindfulness que está sempre presente a todo o momento. É o processo fisiológico transversal a todas as outras técnicas e sistemas mindful, que quando realizado de forma mais lenta e profunda, permite a obtenção de benefícios na saúde ao nível respiratório, cardiovascular, cardio respiratório e nervoso. (Russo, Santarelli \& Rourke, 2017) alavancando, ampliando e otimizando os benefícios e efeitos sentidos durante a prática do Mindfulness.

Existem programas desenhados com foco em várias das técnicas de mindfulness, como o Mindfulness Based Stress Reduction (MBSR), têm o potencial de reduzir a preocupação e melhorar as funções cognitivas (Lenze, Hickman, Hershey, Wendleton, Ly, Dixon, Doré, Wetherell, 2014).

Tabela 2. Programa MBSR

0 programa tem 8 semanas, com uma sessão semanal de 2 horas e meia, e um dia intensivo. É fundamentalmente prático com exercícios de grupo e meditação mindfulness guiada, incluindo body-scan, respiração consciente e meditações sentadas. 
https://doi.org/10.29073/jim.v2i2.426

\begin{tabular}{|c|l|}
\hline Sessão $\mathrm{n}^{0}$ & Temas e Atividades \\
\hline 1 & Introdução ao Mindfulness \\
\hline 2 & Perceção \\
\hline 3 & Mindfulness da respiração e o corpo em movimento \\
\hline 4 & Aprender sobre os padrões de reação ao stress \\
\hline 5 & $\begin{array}{l}\text { Aprender a lidar com o stress: usar o mindfulness para responder em vez } \\
\text { de reagir }\end{array}$ \\
\hline 6 & \begin{tabular}{l} 
Comunicações stressantes e mindfulness interpessoal \\
\hline 7
\end{tabular} \\
\hline 8 & \begin{tabular}{l} 
Como tomar melhor conta de mim \\
\hline Dia Intensivo de mindfulness com práticas de meditação mindfulness.
\end{tabular} \\
\hline
\end{tabular}

Fonte: Elaboração própria adaptado de Full Catastrophe Living, how to cope with stress, pain and illness using mindfulness meditation, 2013, by Jon Kebat-Zinn

O MBSR consiste assim numa articulação sinérgica de meditação mindfulness, consciência corporal e movimento consciente, com o objectivo de ajudar a desenvolver a atenção plena e auto-consciência (Raj and Kumar, 2018).

Técnicas como o mindfulness, o reforço do humor e das emoções positivas, diminuem o domínio das emoções negativas na mente e no corpo e melhoram a relação profissional-paciente, pelo aumento da resiliência individual, pessoal e institucional (Vaz de Almeida, Rodrigues, P., Rodrigues, M., Pinheiro \& Nunes, 2021).

\subsection{Os benefícios Bio-Psico-Sociais do Mindfulness na pessoa idosa}

Sendo o Mindfulness uma forma de prática e treino, para indivíduos com problemas psicológicos e emocionais devido a eventos de vida, condições físicas, dor física , sintomas de stress e ansiedade bem sentimentos mais constrangedores, pode ser usado para promover o bem-estar entre os participantes, e é benéfico na promoção do tratamento agudo de um distúrbio (Raj and Kumar, 2018), bem como atua 
na melhoria da condição de pacientes que sofrem de doenças crónicas e ajuda-os a lidarem com uma ampla variedade de problemas clínicos (Niazi e Niazi, 2011). Há evidências de que pacientes com doenças crónicas como diabetes, hipertensão, cancro, distúrbios imunológicos, dor crónica, distúrbios do sono, dores nas costas, depressão clínica, stress e ansiedade, podem beneficiar do MBSR. Uma vantagem do MBSR é o de apresentar poucos riscos e poder aumentar a capacidade dos pacientes de controlar a dor, alterações de humor e de vida, além de melhorar a qualidade de vida (Niazi e Niazi, 2011).

Em séniores, segundo Ublosakka-Jones, Tongdee, Pachirat, \& Jones (2018), o treino da respiração lenta e consciente - mindful - não é apenas eficaz na redução da tensão arterial em repouso, ou mesmo em idosos com hipertensão sistólica bem controlada, aumenta também a força muscular inspiratória, a capacidade pulmonar e a duração do exercício com os braços. Por outro lado, Noble \& Hochman (2019), afirmam que os processos neurofisiológicos ativados durante a respiração lenta e profunda aumentam os resultados terapêuticos cognitivos e comportamentais, obtidos por meio de várias práticas de movimento consciente mente-corpo.

As técnicas de respiração como as utilizadas nos programas de Redução do Stress baseados em Mindfulness ou nas práticas de sistemas de exercícios com sequências específicas de movimentos tais como o Yoga, Qi Gong e o Tai Ji, são regularmente recomendadas para relaxamento, controle do stress, ativar estados psicofisiológicos para melhorar o funcionamento dos órgãos ( Zope, A., \& Zope, R. A., 2013). Uma revisão sistemática que analisou 15 estudos apoiou os efeitos positivos das intervenções de relaxamento na depressão e ansiedade entre adultos mais velhos ( Klainin-Yobas, Suzanne Yew , 2015).

Assim e porque quando as pessoas relaxam e se sentem melhores ficam mentalmente menos deprimidas e ansiosas, a sua disponibilidade cognitiva aumenta, compreendem melhor a informação, orientações e instruções em saúde, por exemplo, conseguem assim tomar decisões mais informadas, contribuindo para um envelhecimento mais saudável, sendo que aqui podemos também constatar, que a prática do mindfulness com foco na respiração mais lenta e profunda, pode contribuir para o aumento da literacia em saúde da população sénior e em simultâneo, quando praticada também pelos profissionais de saúde que a assistem, contribui para comunicar de forma emocionalmente mais calma, (Dick et al., 2018), acessível e compreensível uma vez que a respiração, segundo o mesmo autor "fornece a base para a comunicação da emoção e é uma ferramenta para modular emoções e o estado emocional". Desta forma o profissional conseguirá transmitir a informação de saúde de forma mais ACP - Assertiva, Clara e Positiva (Vaz de Almeida, 2020). Existindo uma relação terapêutica mais efectiva e ACP em que 0 profissional de saúde se faz entender e explicar de forma assertiva, clara e positiva e a pessoa sénior, 
https://doi.org/10.29073/jim.v2i2.426

por sua vez, também se consegue explicar melhor, compreender o que é dito, e tomar decisões mais informadas e acertadas em saúde. Será possível de antever também nesta dimensão um processo de envelhecimento mais ativo e saudável.

Fountain-Zaragoza, Londerée, Whitmoye and Prakash, em 2016, por sua vez, na sua investigação, propuseram que o treino da atenção plena é adequado como ferramenta de reabilitação com benefícios cognitivos e emocionais para adultos mais velhos. 0 mindfulness promove 0 foco de atenção e pode mitigar o declínio das habilidades de controle da atenção atrasando o seu desenvolvimento, e permitir que os idosos mantenham as suas habilidades de regulação emocional preservadas (Fountain-Zaragoza, Londeree, Whitmoye and Prakash, 2016).

Em 2017, Fountain-Zaragozaand Prakash, dois dos mesmos autores, agora com mais evidências apontam para melhorias no controle da atenção, de bem-estar psicológico e físico, e consequentes melhorias na inflamação sistêmica, após o treino de mindfulness em adultos mais velhos (FountainZaragoza, Prakash 2017).

Num estudo transversal que comparou o desempenho cognitivo de meditadores e não meditadores na faixa etária geriátrica os meditadores de longo prazo têm habilidades cognitivas superiores do que os não meditadores do grupo de idosos (Prakash, Rastogi, Dubey, Abhishek, Chaudhury, Small, 2012).

As intervenções de meditação para adultos mais velhos são viáveis e a meditação pode ser capaz de compensar o declínio cognitivo normal relacionado à idade ou até mesmo melhorar a função cognitiva em adultos mais velhos (Gard, Hölzel, Lazar, 2014).

A influência do treino de meditação mindfulness foi também estudada no controle executivo e na regulação emocional em idosos, por meio de mudanças comportamentais e eletrofisiológicas (Malinowski, Moore, Mead, Gruber, 2017).

Num estudo, 57 participantes, de 55 a 75 anos de idade, foram alocados aleatoriamente num grupo de treino de respiração consciente, de 8 semanas, ou num grupo de controle de exercícios de treino cerebral. Os resultados mostram que o envolvimento em pouco mais de 10 minutos de prática de mindfulness, cinco vezes por semana, resultou em melhorias significativas nas medidas comportamentais e eletrofisiológicas relacionadas ao desempenho geral de tarefas (Malinowski, Moore, Mead, Gruber, 2017).

Nessa população de idosos com transtornos de stress e dificuldades neuro cognitivas, uma intervenção de atenção plena melhora os resultados clínicos, como preocupação excessiva e depressão, e pode incluir algumas formas de memória imediata. 
Sabemos que com o aumento da idade se pode constatar um aumento da prevalência e incidência de doenças crónicas, e que as mesmas estão associadas a uma série de consequências psicológicas e físicas desconfortáveis. Também as intervenções baseadas em mindfulness, nomeadamente os programas de Mindfulness Based Stress Reduction (MBSR) ou Terapia Cognitiva Baseada em Mindfulness (MBCT), foram conduzidos com benefícios em adultos mais velhos (Hazlett-Stevens, Singer, Chong, 2019). Os resultados apoiaram o uso de MBSR para dor lombar crônica, insónia crónica, melhora da qualidade do sono, aumento do afeto positivo, redução dos sintomas de ansiedade, depressão, melhora da memória e funcionamento operatório, em adultos mais velhos. E o MBCT reduziu efetivamente os sintomas de ansiedade numa amostra de adultos séniores com ansiedade elevada (Hazlett-Stevens, Singer, Chong, 2019).

Numa revisão de 15 artigos os diagnósticos clínicos dos participantes incluíram fibromialgia, dor crónica, artrite reumatóide, diabetes tipo 2 , síndrome da fadiga crónica, sensibilidade química múltipla e diagnósticos cardiovasculares. Segundo Merkes M. (2010), em todos os 15 estudos descobriram que a participação num programa MBSR resultou em melhoria, sendo que nenhuma mudança negativa foi relatada entre o início da participação no programa e o "Follow.up", sendo indicador de manutenção de benefícios a médio-longo prazo. Segundo o estudo liderado por Wang, C., Schmid (2018) a intervenção com a prática mindful Tai Ji pode ser considerada uma opção terapêutica no tratamento multidisciplinar da fibromialgia, uma doença crónica que tem início antes da idade sénior, mas que como doença crónica, tende a prevalecer e a agravar com a idade.

0 programa MBSR já tinha sido provado como tratamento benéfico para a insónia crónica em adultos com 75 anos ou mais (Zhang, Liu, Xie, Zhao, Shan, Zhang, Kong, Cui, 2015), e para adultos mais velhos que sofrem de sintomas clínicos de preocupação e disfunção cognitiva concomitante (Lenze, Hickman, Hershey, Wendleton, Ly, Dixon, Doré, Wetherell, 2014). Esses são problemas comuns na população sénior, portanto, a intervenção de redução de stress para idosos pode significar ganhos em saúde e ter grande valor para a saúde pública (Lenze, Hickman, Hershey, Wendleton, Ly, Dixon, Doré, Wetherell, 2014), e será uma intervenção promissora para a população sénior com uma variedade de problemas de saúde e declínio cognitivo. 0 MBCT pode reduzir a ansiedade geriátrica, embora os seus efeitos na depressão geriátrica não tenham sido medidos (Hazlett-Stevens, Singer, Chong, 2019).

No geral, os resultados indicam que o envolvimento no treino de meditação melhora a manutenção da atenção visão espacial direcionada a um objetivo, e pode ser uma estratégia útil para neutralizar o declínio cognitivo associado ao envelhecimento, mas os estudos não encontram as melhorias específicas esperadas no controle executivo e regulação emocional (Malinowski, Moore, Mead, Gruber, 2017). 
https://doi.org/10.29073/jim.v2i2.426

Também nos sintomas motores e não motores que acompanham a doença de Parkinson as práticas mindful de Tai Ji e Qi Gong, parecem favorecer a qualidade de vida, a função motora e o humor (Song et al, 2017). Outra doença crónica que tende a agravar com a idade. Na manutenção da função e boa saúde da função cardíaca, estas práticas parecem também hipertensão, pressão arterial sistólica e diastólica e frequência cardíaca em repouso.

Para os programas de MBSR e MBCT são necessários mais ensaios clínicos adicionais em grande escala conduzidos com adultos mais velhos que lidam com a variedade de desafios físicos, comportamentais e cognitivos que os idosos comumente enfrentam (Hazlett-Stevens, Singer, Chong, 2019).

Tabela 3. Benefícios do Mindfulness nas dimensões Bio - Psico - Social da pessoa idosa

\begin{tabular}{|c|c|c|}
\hline $\begin{array}{c}\text { BI0 } \\
\text { Bem-estar Físico }\end{array}$ & $\begin{array}{c}\text { PSICO } \\
\text { Cognitivos e emocionais, } \\
\text { bem-estar psicológico }\end{array}$ & $\begin{array}{c}\text { SOCIAL } \\
\text { Desempenho, autonomia e relação } \\
\text { com os outros }\end{array}$ \\
\hline $\begin{array}{l}\text { Melhora a qualidade do } \\
\text { sono. (Hazlett-Stevens, } \\
\text { Singer, Chong, 2019) }\end{array}$ & $\begin{array}{l}\text { Melhora a regulação emocional na } \\
\text { gestão da preocupação excessiva e } \\
\text { depressão e memória imediata } \\
\text { (Malinowski, Moore, Mead,Gruber, } \\
\text { 2017). }\end{array}$ & $\begin{array}{l}\text { Melhora a relação profissional- } \\
\text { paciente, pelo aumento da resiliência } \\
\text { individual, pessoal e institucional } \\
\text { (Vaz de Almeida, Rodrigues, P., } \\
\text { Rodrigues, M., Pinheiro \& Nunes, } \\
\text { 2021). } \\
\text { Contribui para uma comunicação, } \\
\text { relação terapêutica e com os outros } \\
\text { mais ACP - Assertiva, Clara e Positiva } \\
\text { (Vaz de almeida, } 2020 \text { contribuindo } \\
\text { para a melhoria da literacia em saúde } \\
\text { no envelhecimento. }\end{array}$ \\
\hline $\begin{array}{l}\text { Dor lombar crônica, } \\
\text { insônia crônica (Hazlett- } \\
\text { Stevens, Singer, Chong, } \\
\text { 2019). }\end{array}$ & $\begin{array}{l}\text { Atua em transtornos de stress e } \\
\text { dificuldades neurocognitivas } \\
\text { melhorando os resultados clínicos } \\
\text { tais como, preocupação excessiva } \\
\text { e depressão, e pode incluir } \\
\text { algumas formas de desempenho } \\
\text { imediato da memória (Wetherell, } \\
\text { J. L., Hershey, T., Hickman, S., } \\
\text { 2017) }\end{array}$ & $\begin{array}{l}\text { Aumenta o afeto positivo, reduz os } \\
\text { sintomas de ansiedade e depressão } \\
\text { (Hazlett-Stevens, Singer, Chong, } \\
2019 \\
\text { Redução de quedas (Li, F., Harmer et } \\
\text { al (2019) }\end{array}$ \\
\hline
\end{tabular}




\begin{tabular}{|c|c|c|}
\hline $\begin{array}{l}\text { Fibromialgia, dor crónica, } \\
\text { artrite reumatóide, } \\
\text { diabetes tipo } 2 \text { síndrome } \\
\text { da fadiga crônica, } \\
\text { sensibilidade química } \\
\text { múltipla e patologias } \\
\text { cardiovasculares (Merkes } \\
\text { M., 2010); Wang, C., } \\
\text { Schmid, C. H., Fielding, T. } \\
\text { et al (2018) }\end{array}$ & $\begin{array}{l}\text { Ativa regiões do cérebro } \\
\text { envolvidas na consciência } \\
\text { corporal, atenção e integração da } \\
\text { emoção e do processamento } \\
\text { sensorial. (Acevedo, B., P., } \\
\text { Pospos, S., \& Lavretsky, H., } \\
2016 \text { ) }\end{array}$ & $\begin{array}{l}\text { comunicar de forma emocionalmente } \\
\text { mais calma e compreensível (Dick et } \\
\text { al., 2018) } \\
\text { Função motora, qualidade de vida } \\
\text { humor na doença de Parkinson } \\
\text { (Song, R., Grabowska, W., Park, M., } \\
\text { et al, 2017) }\end{array}$ \\
\hline $\begin{array}{l}\text { Promove melhorias na } \\
\text { inflamação sistémica, } \\
\text { (Fountain-Zaragoza, } \\
\text { Prakash,2017). }\end{array}$ & $\begin{array}{l}\text { Promove o foco de atenção. Pode } \\
\text { mitigar o declínio das habilidades } \\
\text { de controlo da atenção.Estimula a } \\
\text { auto-regulaçãor emocional } \\
\text { (Fountain-Zaragoza, Londeree, } \\
\text { Whitmoye and Prakash, 2016). }\end{array}$ & $\begin{array}{l}\text { Melhora o desempenho geral de } \\
\text { tarefas (Malinowski, Moore, Mead, } \\
\text { Gruber, 2017). }\end{array}$ \\
\hline $\begin{array}{l}\text { Controlo da função } \\
\text { cardíaca, (Zou etc. al } \\
\text { 2017; Liu et al., 2014) }\end{array}$ & $\begin{array}{l}\text { Reduz a preocupação e disfunção } \\
\text { cognitiva concomitante (Lenze, } \\
\text { Hickman, Hershey, Wendleton, } \\
\text { Ly, Dixon, Doré, Wetherell, 2014). } \\
\text { Relaxamento, controlo do stress } \\
\text { (Zope,A \& Zope, R.A, 2013) }\end{array}$ & $\begin{array}{l}\text { Melhora a função motora e } \\
\text { diminuição do risco de queda (Song, } \\
\text { R., Grabowska, W., Park, M., et al, } \\
\text { 2017) }\end{array}$ \\
\hline
\end{tabular}

Fonte: Elaboração própria com base nos autores citados

Como evidenciado no estudo "Bem-estar, Mindfulness e Saúde Oral: benefícios para os profissionais e pacientes" outro aspecto benéfico importante do mindfulness, e dos programas baseados em mindfulness, que importa referir é a nível da saúde oral. Quer para os pacientes que sofrem com medo e ansiedade, como para os profissionais de saúde oral - dentistas, higienistas orais, assistentes dentários - que relatam altos níveis de stress, um diminuto bem-estar e um maior burnout (Vaz de Almeida et al, 2021). Aumentar a capacidade dos pacientes de gerir emoções e dotar o profissional de saúde com mais competências para investir na relação, é um dos passos para melhorar o bem-estar na saúde oral (Vaz de Almeida et al, 2021). Para o paciente, a compreensão da importância de se preparar para a consulta, conseguindo, através de um processo de relaxamento, respiração, pensamentos positivos, enfrentar os seus medos, melhora os resultados de saúde tanto biológicos como psicossociais (Vaz de Almeida et al, 2021).

0 maior conhecimento e compreensão de ambos os intervenientes, dos medos e ansiedades provocadas pelo encontro em saúde dentária, pode eventualmente melhorar a consciência coletiva de que paciente 
https://doi.org/10.29073/jim.v2i2.426

e profissional sofrem na interação, embora de formas diversas (Vaz de Almeida et al, 2021) e no nosso entendimento, por consequência, irá contribuir para um envelhecimento mais saudável para ambos.

\section{RESULTADOS E DISCUSSÃO}

A pesquisa realizada com base nas 33 referências bibliográficas consultadas num total de 129 autores e acesso a 130 estudos, permite concluir que o Mindfulness, técnicas, sistemas e programas associados, têm vindo a ser desenvolvidos e aplicados junto de pessoas com mais de 55 anos, tanto no Oriente como no Ocidente. No Oriente fazendo parte integrante da cultura indiana e chinesa, através da prática de sistemas constituídos por sequências de exercícios tais como o Yoga, o Qi Gong e o Tai Ji, com vista à promoção e educação para a saúde, à manutenção da saúde e do bem-estar geral. Integra todo um sistema holístico de cultivo da saúde e da longevidade, focando em dar mais qualidade à vida, saúde e bem-estar, aos anos e não apenas mais anos à vida, conduzindo a um envelhecimento ativo, autónomo, saudável e sustentável. No ocidente estas práticas mindful orientais têm criado raízes, praticantes e seguidores desde há mais de 30 anos. Pela pesquisa efectuada, o "Mindfulness", com esta designação foi introduzido por Jon Kabat-Zinn que fundou e desenvolveu o programa Mindfulness Based Stress Reduction (MBSR) na década de 1970 no Centro Médico da Universidade de Massachusetts.

A pesquisa demonstrou que é possível à pessoa idosa utilizadora regular destas práticas beneficiar dos seus efeitos a curto, médio e longo prazo, nas dimensões Bio - Psico - Social. o MBSR é uma abordagem segura, eficaz e integrativa para redução de stress e ansiedade que acompanham a vida diária e as doenças crónicas. Foi considerado que os profissionais de saúde podem usar esta intervenção com segurança e eficácia numa variedade de populações de pacientes (Praissman, 2008) e que os profissionais que lidam diariamente com pacientes podem também beneficiar dos seus efeitos positivos.

\section{CONCLUSÕES, LIMITAÇÕES, RECOMENDAÇÕES DE NATUREZA ACADÉMICA E PRÁTICA}

Após a pesquisa realizada é possível concluir que o Mindfulness, técnicas, práticas e sistemas mindful, contribuem para o "processo de desenvolvimento e manutenção da capacidade funcional que permite 0 bem-estar em idade avançada" definição de envelhecimento saudável segundo WHO (2015, p.13).

A ciência sendo um sistema dinâmico e evolutivo, dada a importância e pertinência desta área de intervenção e investigação permite e sugere a inclusão de futuros estudos. Contudo, pelos artigos e 
https://doi.org/10.29073/jim.v2i2.426

autores consultados, é possível concluir que faz todo o sentido a recomendação e implementação das práticas mindful em planos de atividades e programas de instituições, organismos e entidades vocacionados e direcionados para a educação da saúde, promoção da saúde e bem-estar junto da população acima dos 55 anos.

Será importante para pesquisas futuras incorporar grupos de comparação controlados por placebo para estabelecer claramente o papel causal das práticas de mindfulness na promoção da saúde holística em adultos mais velhos. (Fountain-Zaragoza, Prakash 2017).

Numa perspectiva económica, as intervenções mente-corpo baseadas em mindfulness, como o MBSR, reduzem o stress e aumentam a resiliência, têm o potencial de reduzir substancialmente a utilização de serviços de saúde a um custo relativamente baixo e, portanto, podem servir como componentes-chave em qualquer sistema de saúde da população e de prestação de cuidados de saúde (Stahl et al, 2015).

São consideradas práticas seguras, bem recebidas, de baixo custo benefícios reais e evidentes para a saúde Bio-Psico-Social da população em geral com efeitos bem estudados e mais direcionadas à população sénior. 
https://doi.org/10.29073/jim.v2i2.426

\section{BIBLIOGRAFIA}

Almeida, C.V. (2020) Literacia em saúde e capacitação dos profissionais de saúde: o modelo de comunicação em saúde ACP. In XIV Jornadas APDIS (30 outubro 2020, p. 49). Apresentação selecionada para Prémio Lucília Paiva. Disponível em: Cristina Vaz Almeida.pdf (rcaap.pt)

Acevedo, B. P., Pospos, S., \& Lavretsky, H. (2016). The Neural Mechanisms of Meditative Practices: Novel Approaches for Healthy Aging. Current behavioral neuroscience reports, 3(4), 328-339. https://doi.org/10.1007/s40473-016-0098-x

Bishop, S. R., Lau, M., Shapiro, S., Carlson, L., Anderson, N. D., Carmody, J., Segal, Z. V., Abbey, S., Speca, M., Velting, D., \& Devins, G. (2004). Mindfulness: A proposed operational definition. Clinical Psychology: Science and Practice, 11(3), 230-241. https://doi.org/10.1093/clipsy.bph077

Davids, T. \& Stede, W. (Eds.) (1921/2001). Pali-English Dictionary. New Delhi, India: Munshiram Manoharlal Publishers Pvt, Ltd.

Dick, T. E., Dutschmann, M., Feldman, J. L., Fong, A. Y., Hülsmann, S., Morris, K. M., Ramirez, J. M., Smith, J. C., \& Respiratory Neurobiology Consortium (2018). Facts and challenges in respiratory neurobiology. Respiratory physiology \& neurobiology, 258, 104-107. https://doi.org/10.1016/j.resp.2015.01.014

Fountain-Zaragoza, S., \& Prakash, R. S. (2017). Mindfulness Training for Healthy Aging: Impact on Attention, Well-Being, and Inflammation. Frontiers in aging neuroscience, 9, 11. https://doi.org/10.3389/fnagi.2017.00011

Gard, T., Hölzel, B. K., \& Lazar, S. W. (2014). The potential effects of meditation on age-related cognitive decline: a systematic review. Annals of the New York Academy of Sciences, 1307, 89-103. https://doi.org/10.1111/nyas.12348

Germer, C. (2004). What is mindfulness? Insight Journal, 22(3), 24-29. Retrieved from http://www.mindfulselfcompassion.org/articles/insight germermindfulness.pdf

Hazlett-Stevens, H., Singer, J., \& Chong, A. (2019). Mindfulness-Based Stress Reduction and Mindfulness-Based Cognitive Therapy with Older Adults: A Qualitative Review of Randomized Controlled Outcome Research. Clinical gerontologist, 42(4), 347-358. https://doi.org/10.1080/07317115.2018.1518282

Kabat-Zinn, J. (2003). Mindfulness-based interventions in context: Past, present, and future. Clinical Psychology: Science and Practice, 10(2). 144-156.

Kabat-Zinn, J. (2013). Full Catastrophe Living : How to Cope With Stress, Pain and Illness Using Mindfulness Meditation. New York: Bantam Books. Paperback Edition.

Kebat-Zinn, J. (2013. Full Catastrophe Living, how to cope with stress, pain and illness using mindfulness meditation 
Klainin-Yobas, P., Oo, W. N., Suzanne Yew, P. Y., \& Lau, Y. (2015). Effects of relaxation interventions on depression and anxiety among older adults: a systematic review. Aging \& mental health, 19(12), 1043-1055. https://doi.org/10.1080/13607863.2014.997191

Lenze, E. J., Hickman, S., Hershey, T., Wendleton, L., Ly, K., Dixon, D., Doré, P., \& Wetherell, J. L. (2014). Mindfulness-based stress reduction for older adults with worry symptoms and co-occurring cognitive dysfunction. International journal of geriatric psychiatry, 29(10), 991-1000. https://doi.org/10.1002/gps.4086

Li, F., Harmer, P., Eckstrom, E., Fitzgerald, K., Chou, L. S., \& Liu, Y. (2019). Effectiveness of Tai Ji Quan vs Multimodal and Stretching Exercise Interventions for Reducing Injurious Falls in Older Adults at High Risk of Falling: Follow-up Analysis of a Randomized Clinical Trial. JAMA network open, 2(2), e188280. https://doi.org/10.1001/jamanetworkopen.2018.8280

Li, F., Harmer, P., Fisher, K. J., McAuley, E., Chaumeton, N., Eckstrom, E., \& Wilson, N. L. (2005). Tai Chi and fall reductions in older adults: a randomized controlled trial. The journals of gerontology. Series A, Biological sciences and medical sciences, 60(2), 187-194. https://doi.org/10.1093/gerona/60.2.187

Malinowski, P., Moore, A. W., Mead, B. R., \& Gruber, T. (2017). Mindful Aging: The Effects of Regular Brief Mindfulness Practice on Electrophysiological Markers of Cognitive and Affective Processing in Older Adults. Mindfulness, 8(1), 78-94. https://doi.org/10.1007/s12671-015-0482-8

Merkes M. (2010). Mindfulness-based stress reduction for people with chronic diseases. Australian journal of primary health, 16(3), 200-210. https://doi.org/10.1071/PY09063

Niazi, A. K., \& Niazi, S. K. (2011). Mindfulness-based stress reduction: a non-pharmacological approach for chronic illnesses. North American journal of medical sciences, 3(1), 20-23. https://doi.org/10.4297/najms.2011.320

Nilsson, H., Bülow, P. H., \& Kazemi, A. (2015). Mindful Sustainable Aging: Advancing a Comprehensive Approach to the Challenges and Opportunities of Old Age. Europe's journal of psychology, 11(3), 494-508. https://doi.org/10.5964/ejop.v11i3.949

Noble, D. J., \& Hochman, S. (2019). Hypothesis: Pulmonary Afferent Activity Patterns During Slow, Deep Breathing Contribute to the Neural Induction of Physiological Relaxation. Frontiers in physiology, 10, 1176. https://doi.org/10.3389/fphys.2019.01176

Praissman S. (2008). Mindfulness-based stress reduction: a literature review and clinician's guide. Journal of the American Academy of Nurse Practitioners, 20(4), 212-216. https://doi.org/10.1111/j.1745-7599.2008.00306.x

Prakash, R., Rastogi, P., Dubey, I., Abhishek, P., Chaudhury, S., \& Small, B. J. (2012). Long-term concentrative meditation and cognitive performance among older adults. Neuropsychology, development, and cognition. Section B, Aging, neuropsychology and cognition, 19(4), 479494. https://doi.org/10.1080/13825585.2011.630932

Raj, A., Kumar, P. (2018). Efficacy of Mindfulness Based Stress Reduction (MBSR) :A Brief Overiew. Journal of Disability Management and Rehabilitation. 4(1). 
https://doi.org/10.29073/jim.v2i2.426

https://www.researchgate.net/publication/328540330 Efficacy of Mindfulness Based Str ess Reduction MBSR A Brief Overiew

Russo, M. A., Santarelli, D. M., \& O'Rourke, D. (2017). The physiological effects of slow breathing in the healthy human. Breathe (Sheffield, England), 13(4), 298-309. https://doi.org/10.1183/20734735.009817

Song, R., Grabowska, W., Park, M., Osypiuk, K., Vergara-Diaz, G. P., Bonato, P., Hausdorff, J. M., Fox, M., Sudarsky, L. R., Macklin, E., \& Wayne, P. M. (2017). The impact of Tai Chi and Qigong mind-body exercises on motor and non-motor function and quality of life in Parkinson's disease: A systematic review and meta-analysis. Parkinsonism \& related disorders, 41, 3-13. https://doi.org/10.1016/j.parkreldis.2017.05.019

Stahl JE, Dossett ML, LaJoie AS, Denninger JW, Mehta DH, Goldman R, et al. (2015) Relaxation Response and Resiliency Training and Its Effect on Healthcare Resource Utilization. PLoS ONE 10(10): e0140212.https://doi.org/10.1371/journal.pone.0140212

Vaz de Almeida, C., Rodrigues, P., Rodrigues, M., Pinheiro, D. \& Nunes, C. (2021). Bem-Estar, Mindfulness e Saúde Oral: Benefícios para Profissionais e Pacientes. Lisboa: APPSP. DOI: 10.5281/zenodo.455743 (no prelo)

Wang, C., Schmid, C. H., Fielding, R. A., Harvey, W. F., Reid, K. F., Price, L. L., Driban, J. B., Kalish, R., Rones, R., \& McAlindon, T. (2018). Effect of tai chi versus aerobic exercise for fibromyalgia: comparative effectiveness randomized controlled trial. BMJ (Clinical research ed.), 360, k851. https://doi.org/10.1136/bmj.k851

Wetherell, J. L., Hershey, T., Hickman, S., Tate, S. R., Dixon, D., Bower, E. S., \& Lenze, E. J. (2017). Mindfulness-Based Stress Reduction for Older Adults With Stress Disorders and Neurocognitive Difficulties: A Randomized Controlled Trial. The Journal of clinical psychiatry, 78(7), e734-e743. https://doi.org/10.4088/JCP.16m10947

Zhang, J. X., Liu, X. H., Xie, X. H., Zhao, D., Shan, M. S., Zhang, X. L., Kong, X. M., \& Cui, H. (2015). Mindfulness-based stress reduction for chronic insomnia in adults older than 75 years: a randomized, controlled, single-blind clinical trial. Explore (New York, N.Y.), 11(3), 180-185. https://doi.org/10.1016/j.explore.2015.02.005

Zope, S. A., \& Zope, R. A. (2013). Sudarshan kriya yoga: Breathing for health. International journal of yoga, 6(1), 4-10. https://doi.org/10.4103/0973-6131.105935

Zou, L., SasaKi, J. E., Wang, H., Xiao, Z., Fang, Q., \& Zhang, M. (2017). A Systematic Review and Meta-Analysis Baduanjin Qigong for Health Benefits: Randomized Controlled Trials. Evidence-based complementary and alternative medicine: eCAM, 2017, 4548706. https://doi.org/10.1155/2017/4548706 